\title{
Computational Studies of the Tropone Natural Products, Thiotropocin, Tropodithietic acid, and Troposulfenin. Significance of Thiocarbonyl-enol Tautomerism
}

Edyta M. Greer, ${ }^{\text {a David Aebisher, }}{ }^{\mathbf{a}}$ Alexander Greer, ${ }^{\mathbf{a},{ }^{*}}$ and Ronald Bentley ${ }^{\mathbf{b}}$,

${ }^{a}$ Department of Chemistry and Graduate Center \& The City University of New York (CUNY), Brooklyn College, Brooklyn, NY 11210

${ }^{\mathrm{b}}$ Department of Biological Sciences, University of Pittsburgh, Pittsburgh, PA 15260

Email: agreer@brooklyn.cuny.edu; rbentley@pitt.edu

Supporting Materials

Table of Contents

Page

S1 Table of Contents.

S2 List of authors for reference 25.

S2 Computed geometry of the stationary point of thiotropocin $\mathbf{1}$ and energetics.

S3 Computed geometry of the stationary point of tropodithietic acid $\mathbf{2}$ and energetics.

S3 Computed geometry of the stationary point of troposulfenin $\mathbf{3}$ and energetics.

S4 Computed geometry of the stationary point of Conjugate Base $\mathbf{4}$ and energetics.

S4 Computed geometry of the stationary point of TS12 and energetics.

S5 Computed geometry of the stationary point of $\mathbf{1}+\mathbf{H}_{2} \mathbf{O}$ and energetics.

S5 Computed geometry of the stationary point of TS12 $+\mathbf{H}_{2} \mathrm{O}$ and energetics.

S6 Computed geometry of the stationary point of $\mathbf{2}+\mathbf{H}_{2} \mathbf{O}$ and energetics.

S7 Computed geometry of the stationary point of $\mathbf{5}+\mathbf{H}_{2} \mathbf{O}$ and energetics.

S7 Computed geometry of the stationary point of TS56 $+\mathbf{H}_{2} \mathrm{O}$ and energetics.

S8 Computed geometry of the stationary point of $6+\mathbf{H}_{2} \mathrm{O}$ and energetics.

S9 Computed geometry of the stationary point of TS67 $+\mathbf{H}_{2} \mathrm{O}$ and energetics.

S9 Computed geometry of the stationary point of $7+\mathbf{H}_{2} \mathrm{O}$ and energetics.

S10 Computed geometry of the stationary point of TS73+ $\mathbf{H}_{2} \mathbf{O}$ and energetics.

S10 Computed geometry of the stationary point of $\mathbf{3}+\mathbf{H}_{2} \mathbf{O}$ and energetics.

S11 Table S1. Computed Entropies, Enthalpies, and Free Energies for 1, 2, and TS12. 


\section{Complete list of authors for reference 25}

Frisch, M. J., Trucks, G. W., Schlegel, H. B., Scuseria, G. E., Robb, M. A., Cheeseman, J. R., Montgomery, J. A., Jr., Vreven, T., Kudin, K. N., Burant, J. C., Millam, J. M., Iyengar, S. S., Tomasi, J., Barone, V., Mennucci, B., Cossi, M., Scalmani, G., Rega, N., Petersson, G. A., Nakatsuji, H., Hada, M., Ehara, M., Toyota, K., Fukuda, R., Hasegawa, J., Ishida, M., Nakajima, T., Honda, Y., Kitao, O., Nakai, H., Klene, M., Li, X., Knox, J. E., Hratchian, H. P., Cross, J. B., Bakken, V., Adamo, C., Jaramillo, J., Gomperts, R., Stratmann, R. E., Yazyev, O., Austin, A. J., Cammi, R., Pomelli, C., Ochterski, J. W., Ayala, P. Y., Morokuma, K., Voth, G. A., Salvador, P., Dannenberg, J. J., Zakrzewski, V. G., Dapprich, S., Daniels, A. D., Strain, M. C., Farkas, O., Malick, D. K., Rabuck, A. D., Raghavachari, K., Foresman, J. B., Ortiz, J. V., Cui, Q., Baboul, A. G., Clifford, S., Cioslowski, J., Stefanov, B. B., Liu, G., Liashenko, A., Piskorz, P., Komaromi, I., Martin, R. L., Fox, D. J., Keith, T., Al-Laham, M. A., Peng, C. Y., Nanayakkara, A., Challacombe, M., Gill, P. M. W., Johnson, B., Chen, W., Wong, M. W., Gonzalez, C., and Pople, J. A. Gaussian 03, revision B.05; Gaussian, Inc.: Pittsburgh, PA, 2003.

Geometries, absolute energies, sum of electronic and zero-point energies, sum of electronic and thermal energies, sum of electronic and thermal free energies and the number of imaginary frequencies of the stationary points presented in the manuscript

Thiotropocin 1 (C1)

Absolute energy: -1329.3046

Sum of electronic and zero-point Energies $=-1329.1981$

Sum of electronic and thermal Energies $=-1329.1877$

Sum of electronic and thermal Enthalpies $=-1329.1867$

Sum of electronic and thermal Free Energies $=-1329.2346$

Number of Imaginary frequencies: 0

01

$\begin{array}{lrrr}\mathrm{C} & -0.13273300 & 2.84066000 & 0.00002900 \\ \mathrm{C} & 1.19065100 & 2.40499700 & 0.00001900 \\ \mathrm{C} & 1.70032900 & 1.09140500 & -0.00000800 \\ \mathrm{C} & -1.35244300 & 2.14209100 & 0.00001100 \\ \mathrm{C} & 0.91652500 & -0.09820700 & -0.00002200 \\ \mathrm{C} & -1.53114200 & 0.76021600 & 0.00000200 \\ \mathrm{C} & -0.45716200 & -0.19676300 & 0.00000200 \\ \mathrm{H} & -0.24094300 & 3.92361300 & 0.00004200 \\ \mathrm{H} & 1.96049200 & 3.17213500 & 0.00003500 \\ \mathrm{H} & -2.25617200 & 2.74653500 & -0.00001000 \\ \mathrm{C} & 1.56464800 & -1.44525900 & -0.00000400 \\ \mathrm{O} & 3.02495800 & 0.98058500 & 0.00001900 \\ \mathrm{H} & 3.25949000 & -0.00087900 & -0.00002900 \\ \mathrm{O} & 0.69863300 & -2.42616700 & 0.00005800 \\ \mathrm{O} & 2.79498800 & -1.59035600 & -0.00010000 \\ \mathrm{~S} & -1.13305100 & -1.78984100 & 0.00005400\end{array}$


Tropodithietic acid $2(\mathrm{C} 1)$

Absolute energy: -1329.3069

Sum of electronic and zero-point Energies $=-1329.2006$

Sum of electronic and thermal Energies $=-1329.1902$

Sum of electronic and thermal Enthalpies $=-1329.1892$

Sum of electronic and thermal Free Energies $=-1329.2373$

Number of Imaginary frequencies: 0

01

$\begin{array}{lrrr}\mathrm{C} & -0.12470900 & 2.87246100 & 0.00045200 \\ \mathrm{C} & 1.17089300 & 2.42627000 & 0.00012400 \\ \mathrm{C} & 1.73953000 & 1.08575700 & -0.00020500 \\ \mathrm{C} & -1.35762500 & 2.15425100 & 0.00041900 \\ \mathrm{C} & 0.92672400 & -0.13014900 & 0.00008400 \\ \mathrm{C} & -1.48641300 & 0.79404900 & -0.00016700 \\ \mathrm{C} & -0.44671500 & -0.20532900 & 0.00015300 \\ \mathrm{H} & -0.23835200 & 3.95494800 & 0.00064400 \\ \mathrm{H} & 1.95110600 & 3.18361400 & 0.00003700 \\ \mathrm{H} & -2.26877700 & 2.74835400 & 0.00054100 \\ \mathrm{C} & 1.58306000 & -1.47157100 & 0.00011800 \\ \mathrm{O} & 2.99659400 & 0.98586700 & -0.00060000 \\ \mathrm{~S} & -1.34043400 & -1.70903500 & 0.00020800 \\ \mathrm{~S} & -2.96148100 & -0.17339500 & -0.00047800 \\ \mathrm{O} & 0.87164400 & -2.48453500 & 0.00035100 \\ \mathrm{O} & 2.89606300 & -1.54303400 & 0.00001500 \\ \mathrm{H} & 3.24379100 & -0.58884600 & -0.00090000\end{array}$

Troposulfenin 3 (C1)

Absolute energy: -1329.2693

Sum of electronic and zero-point Energies $=-1329.167057$

Sum of electronic and thermal Energies $=-1329.155835$

Sum of electronic and thermal Enthalpies $=-1329.154890$

Sum of electronic and thermal Free Energies $=-1329.204926$

Number of Imaginary frequencies: 0

01

$\begin{array}{lrrc}\mathrm{C} & -0.43558300 & 2.78030500 & -0.39523700 \\ \mathrm{C} & 0.89798100 & 2.55557700 & -0.26893000 \\ \mathrm{C} & 1.61513800 & 1.34892400 & 0.19769900 \\ \mathrm{C} & -1.54209800 & 1.89057500 & -0.15461500 \\ \mathrm{C} & 0.99276300 & 0.01703300 & 0.03073500 \\ \mathrm{C} & -1.52310400 & 0.54022700 & 0.03806600 \\ \mathrm{C} & -0.34638000 & -0.29600500 & -0.00903600 \\ \mathrm{H} & -0.72951600 & 3.79175600 & -0.66969100 \\ \mathrm{H} & 1.57566800 & 3.38809700 & -0.44558400 \\ \mathrm{H} & -2.52203600 & 2.36149400 & -0.14561100\end{array}$




$\begin{array}{lrrr}\mathrm{C} & 1.85862800 & -1.18232500 & -0.07901800 \\ \mathrm{O} & 2.74415300 & 1.47518200 & 0.66033000 \\ \mathrm{O} & 1.06190100 & -2.33485500 & -0.16538200 \\ \mathrm{O} & 3.05274700 & -1.28767800 & -0.13255100 \\ \mathrm{~S} & -0.60418300 & -2.02867700 & -0.13199700 \\ \mathrm{~S} & -3.10446000 & -0.31896500 & 0.17840100 \\ \mathrm{H} & -2.96030700 & -0.72614800 & 1.46126400\end{array}$

Conjugate Base 4 (C1)

Absolute energy: -1328.7881

Sum of electronic and zero-point Energies $=-1328.695139$

Sum of electronic and thermal Energies $=-1328.684526$

Sum of electronic and thermal Enthalpies $=-1328.683581$

Sum of electronic and thermal Free Energies $=-1328.733391$

Number of Imaginary frequencies: 0

$-11$

$\begin{array}{lrrr}\mathrm{C} & -0.27654200 & 2.84463300 & 0.00014200 \\ \mathrm{C} & 1.05451500 & 2.51247100 & 0.00008400 \\ \mathrm{C} & 1.74792300 & 1.22272300 & -0.00009600 \\ \mathrm{C} & -1.45310500 & 2.04799000 & 0.00007200 \\ \mathrm{C} & 0.98097200 & -0.04561900 & 0.00000000 \\ \mathrm{C} & -1.54489800 & 0.66955100 & -0.00002000 \\ \mathrm{C} & -0.38673000 & -0.21468900 & -0.00001100 \\ \mathrm{H} & -0.47451400 & 3.91810500 & 0.00024700 \\ \mathrm{H} & 1.76751500 & 3.33512900 & 0.00013000 \\ \mathrm{H} & -2.39835800 & 2.58665900 & 0.00011200 \\ \mathrm{C} & 1.69212000 & -1.36203800 & 0.00010400 \\ \mathrm{O} & 2.99017300 & 1.22625700 & -0.00030300 \\ \mathrm{O} & 0.79800500 & -2.39802400 & 0.00004800 \\ \mathrm{O} & 2.88411500 & -1.59278900 & 0.00017600 \\ \mathrm{~S} & -0.91432200 & -1.88446500 & -0.00004300 \\ \mathrm{~S} & -3.03308500 & -0.22638300 & -0.00005100\end{array}$

TS12 (C1) (Figure 2A)

Absolute energy: -1329.3014

Sum of electronic and zero-point Energies $=-1329.1991$

Sum of electronic and thermal Energies $=-1329.1891$

Sum of electronic and thermal Enthalpies $=-1329.1881$

Sum of electronic and thermal Free Energies $=-1329.2352$

Number of Imaginary frequencies: 1

$\begin{array}{lrrr}01 & & & \\ \mathrm{C} & -0.06382500 & 2.85397200 & 0.00014300 \\ \mathrm{C} & 1.23475800 & 2.38352200 & 0.00005000 \\ \mathrm{C} & 1.72980900 & 1.04110200 & -0.00004200 \\ \mathrm{C} & -1.31074900 & 2.17588900 & 0.00017700 \\ \mathrm{C} & 0.90533900 & -0.13382100 & -0.00005500\end{array}$




$\begin{array}{lrrr}\mathrm{C} & -1.49113100 & 0.81132600 & 0.00011900 \\ \mathrm{C} & -0.46519500 & -0.19307900 & 0.00001500 \\ \mathrm{H} & -0.14932200 & 3.93893600 & 0.00020100 \\ \mathrm{H} & 2.02806700 & 3.12685900 & 0.00004400 \\ \mathrm{H} & -2.20171800 & 2.79954000 & 0.00025700 \\ \mathrm{C} & 1.56168100 & -1.48222300 & -0.00015600 \\ \mathrm{O} & 3.01507400 & 0.86805500 & -0.00012000 \\ \mathrm{~S} & -1.31069700 & -1.71218600 & -0.00001500 \\ \mathrm{~S} & -2.97302600 & -0.11845800 & 0.00015400 \\ \mathrm{O} & 0.78525200 & -2.47518600 & -0.00016200 \\ \mathrm{O} & 2.83732600 & -1.51997700 & -0.00022500 \\ \mathrm{H} & 3.15719500 & -0.29828100 & -0.00018400\end{array}$

$\mathbf{1}+\mathbf{H}_{2} \mathbf{O}(\mathrm{C} 1)$ (Figure 2B):

Absolute energy: -1405.7343

Sum of electronic and zero-point Energies $=-1405.6044$

Sum of electronic and thermal Energies $=-1405.5902$

Sum of electronic and thermal Enthalpies $=-1405.5892$

Sum of electronic and thermal Free Energies $=-1405.6464$

Number of Imaginary frequencies: 0

01

$\begin{array}{lrrr}\mathrm{C} & -0.89838000 & 2.82777600 & 0.00304300 \\ \mathrm{C} & 0.46134200 & 2.53146900 & 0.02108200 \\ \mathrm{C} & 1.12158100 & 1.28398600 & 0.02795300 \\ \mathrm{C} & -2.03521400 & 2.00393000 & -0.01344500 \\ \mathrm{C} & 0.47378300 & 0.01274700 & 0.01988100 \\ \mathrm{C} & -2.06076300 & 0.61133200 & -0.01369700 \\ \mathrm{C} & -0.88690700 & -0.22382700 & 0.00286000 \\ \mathrm{H} & -1.11861500 & 3.89364300 & 0.00036100 \\ \mathrm{H} & 1.14465600 & 3.37644700 & 0.02951800 \\ \mathrm{H} & -2.99968900 & 2.50551100 & -0.02686700 \\ \mathrm{C} & 1.24469200 & -1.26863600 & 0.03378900 \\ \mathrm{O} & 2.44534200 & 1.37366900 & 0.04491500 \\ \mathrm{H} & 2.90038400 & 0.48953700 & 0.03029300 \\ \mathrm{O} & 0.46890200 & -2.32778100 & 0.02821400 \\ \mathrm{O} & 2.47715900 & -1.31551100 & 0.05128400 \\ \mathrm{~S} & -1.39477400 & -1.87959100 & 0.00037700 \\ \mathrm{~S} & -3.44126500 & -0.41115900 & -0.03070200 \\ \mathrm{H} & 5.68902600 & -0.09570600 & 0.44464300 \\ \mathrm{O} & 4.94509000 & -0.12787600 & -0.17465200 \\ \mathrm{H} & 4.54812700 & -1.01009600 & -0.07962800\end{array}$

TS12 $+\mathbf{H}_{2} \mathbf{O}(\mathrm{C} 1)$ (Figure 2B):

Absolute energy: -1405.7176

Sum of electronic and zero-point Energies $=-1405.5913$

Sum of electronic and thermal Energies $=-1405.5790$ 
Sum of electronic and thermal Enthalpies $=-1405.5781$

Sum of electronic and thermal Free Energies $=-1405.6313$

Number of Imaginary frequencies: 1

01

$\begin{array}{lrrc}\mathrm{C} & 0.79956300 & 2.85995100 & -0.07554900 \\ \mathrm{C} & -0.53465000 & 2.52945800 & -0.12076200 \\ \mathrm{C} & -1.25079700 & 1.27384500 & -0.07422100 \\ \mathrm{C} & 1.94786400 & 2.04175200 & 0.01859500 \\ \mathrm{C} & -0.58744200 & -0.02427700 & -0.05469400 \\ \mathrm{C} & 1.94818200 & 0.66717300 & 0.05342400 \\ \mathrm{C} & 0.79238300 & -0.20323000 & -0.00469800 \\ \mathrm{H} & 1.00379600 & 3.92866800 & -0.11193300 \\ \mathrm{H} & -1.23135300 & 3.36127200 & -0.18235800 \\ \mathrm{H} & 2.91251800 & 2.54194500 & 0.05417900 \\ \mathrm{C} & -1.27461200 & -1.36724300 & -0.12435700 \\ \mathrm{O} & -2.52173500 & 1.42503900 & -0.07876600 \\ \mathrm{H} & -3.55644300 & 0.66453100 & 0.23687500 \\ \mathrm{O} & -0.45953000 & -2.36034600 & -0.12936500 \\ \mathrm{O} & -2.51250600 & -1.59332400 & -0.20637000 \\ \mathrm{~S} & 1.45229800 & -1.80990100 & -0.00606300 \\ \mathrm{~S} & 3.33872400 & -0.37384200 & 0.13261200 \\ \mathrm{H} & -5.00744300 & -0.09615900 & -0.20255500 \\ \mathrm{O} & -4.29081000 & -0.12461800 & 0.45344300 \\ \mathrm{H} & -3.54372900 & -0.89894500 & 0.16305000\end{array}$

$\mathbf{2}+\mathbf{H}_{2} \mathbf{O}(\mathrm{C} 1)$ (Figure 2B):

Absolute energy: -1405.7407

Sum of electronic and zero-point Energies $=-1405.6101$

Sum of electronic and thermal Energies $=-1405.5962$

Sum of electronic and thermal Enthalpies $=-1405.5952$

Sum of electronic and thermal Free Energies $=-1405.6519$

Number of Imaginary frequencies: 0

01

$\begin{array}{lrrr}\mathrm{C} & 0.81149600 & -2.49592500 & -0.00026400 \\ \mathrm{C} & 1.73633500 & -1.48196800 & -0.00035800 \\ \mathrm{C} & 1.58308500 & -0.03750900 & -0.00042900 \\ \mathrm{C} & -0.61232000 & -2.46180700 & -0.00021800 \\ \mathrm{C} & 0.29189500 & 0.63959300 & -0.00021000 \\ \mathrm{C} & -1.38085700 & -1.33088800 & -0.00007300 \\ \mathrm{C} & -0.95087600 & 0.04339400 & 0.00004100 \\ \mathrm{H} & 1.23561200 & -3.49815300 & -0.00033500 \\ \mathrm{H} & 2.78116600 & -1.78319400 & -0.00049400 \\ \mathrm{H} & -1.12483400 & -3.42134800 & -0.00032600 \\ \mathrm{C} & 0.21643200 & 2.13271000 & -0.00014900 \\ \mathrm{O} & 2.63942300 & 0.66836700 & -0.00072300 \\ \mathrm{H} & 4.33334300 & -0.22509000 & -0.00001800\end{array}$




$\begin{array}{lrrr}\mathrm{O} & -0.89526700 & 2.67461900 & 0.00021400 \\ \mathrm{O} & 1.32954200 & 2.83431800 & -0.00030800 \\ \mathrm{~S} & -2.45859900 & 0.92890900 & 0.00046200 \\ \mathrm{~S} & -3.13912000 & -1.19441000 & 0.00003000 \\ \mathrm{H} & 5.84767800 & -0.60907200 & -0.00041500 \\ \mathrm{O} & 4.95433100 & -0.98144800 & 0.00134600 \\ \mathrm{H} & 2.09518800 & 2.17241900 & -0.00057400\end{array}$

$\mathbf{5}+\mathbf{H}_{2} \mathbf{O}(\mathrm{C} 1)$ (Figure 2C)

Absolute energy: -1405.7284

Sum of electronic and zero-point Energies $=-1405.5978$

Sum of electronic and thermal Energies $=-1405.5839$

Sum of electronic and thermal Enthalpies $=-1405.5829$

Sum of electronic and thermal Free Energies $=-1405.6395$

Number of Imaginary frequencies: 0

$\begin{array}{lrrr}01 & & & \\ \mathrm{C} & -2.45957000 & 2.12536900 & -0.32969300 \\ \mathrm{C} & -1.21672000 & 2.67242400 & -0.20394200 \\ \mathrm{C} & 0.08729100 & 2.09458700 & 0.17908200 \\ \mathrm{C} & -2.87749500 & 0.76127000 & -0.20274700 \\ \mathrm{C} & 0.35480100 & 0.63761600 & 0.05549100 \\ \mathrm{C} & -2.05182100 & -0.30650800 & -0.02511600 \\ \mathrm{C} & -0.60765900 & -0.34636800 & 0.03961300 \\ \mathrm{H} & -3.26461500 & 2.82531300 & -0.54669800 \\ \mathrm{H} & -1.13750200 & 3.75130800 & -0.31789600 \\ \mathrm{H} & -3.94479500 & 0.56643100 & -0.28512100 \\ \mathrm{C} & 1.73508300 & 0.10658300 & 0.01639000 \\ \mathrm{O} & 0.95918100 & 2.86487800 & 0.58423500 \\ \mathrm{~S} & -0.29200800 & -2.07731900 & 0.10163700 \\ \mathrm{~S} & -2.49539100 & -2.02291300 & 0.06129600 \\ \mathrm{O} & 1.91791900 & -1.12686000 & 0.07830200 \\ \mathrm{O} & 2.72850500 & 0.95800800 & -0.14124400 \\ \mathrm{H} & 3.58056300 & 0.44230100 & -0.19624600 \\ \mathrm{H} & 3.94449300 & -1.53161100 & -0.11435300 \\ \mathrm{O} & 4.69825200 & -0.91367600 & -0.18994500 \\ \mathrm{H} & 5.20594000 & -1.17866600 & -0.97184400\end{array}$

TS56 $+\mathbf{H}_{2} \mathrm{O}$ (C1) (Figure 2C)

Absolute energy: -1405.7032

Sum of electronic and zero-point Energies $=-1405.5774$

Sum of electronic and thermal Energies $=-1405.5631$

Sum of electronic and thermal Enthalpies $=-1405.5621$

Sum of electronic and thermal Free Energies $=-1405.6440$

Number of Imaginary frequencies: 1

01

$\begin{array}{llll}\text { C } & -2.33528700 & 2.20207000 & -0.38020900\end{array}$ 


$\begin{array}{lrrc}\mathrm{C} & -1.07793100 & 2.70535200 & -0.22027500 \\ \mathrm{C} & 0.17702200 & 2.07412900 & 0.23124300 \\ \mathrm{C} & -2.80182800 & 0.85533800 & -0.24147800 \\ \mathrm{C} & 0.41299300 & 0.61431900 & 0.06906800 \\ \mathrm{C} & -2.01766100 & -0.23785800 & -0.03202400 \\ \mathrm{C} & -0.57586200 & -0.33865000 & 0.05199500 \\ \mathrm{H} & -3.10840400 & 2.92475000 & -0.63538900 \\ \mathrm{H} & -0.95093200 & 3.77843400 & -0.34595300 \\ \mathrm{H} & -3.87298200 & 0.69506300 & -0.34608400 \\ \mathrm{C} & 1.80839500 & 0.11029900 & -0.00866400 \\ \mathrm{O} & 1.04332600 & 2.79487200 & 0.72653100 \\ \mathrm{~S} & -0.35000500 & -2.09124700 & 0.12811600 \\ \mathrm{~S} & -2.53090000 & -1.93680400 & 0.05772800 \\ \mathrm{O} & 1.95210800 & -1.17386500 & 0.14515000 \\ \mathrm{O} & 2.78607400 & 0.86439200 & -0.27539900 \\ \mathrm{H} & 3.86879600 & 0.01622300 & -0.31874800 \\ \mathrm{H} & 3.24774000 & -1.38882500 & -0.03576600 \\ \mathrm{O} & 4.30907800 & -1.00712200 & -0.22447500 \\ \mathrm{H} & 4.64653300 & -1.31302500 & -1.08395800\end{array}$

6+ $\mathbf{H}_{2} \mathbf{O}(\mathrm{C} 1)$ (Figure 2C)

Absolute energy: -1405.7222

Sum of electronic and zero-point Energies $=-1405.5919$

Sum of electronic and thermal Energies $=-1405.5780$

Sum of electronic and thermal Enthalpies $=-1405.5770$

Sum of electronic and thermal Free Energies $=-1405.6337$

Number of Imaginary frequencies: 0

01

$\begin{array}{lrrc}\mathrm{C} & -2.44250400 & 2.12180400 & -0.38210100 \\ \mathrm{C} & -1.21088500 & 2.67525700 & -0.19060700 \\ \mathrm{C} & 0.05937000 & 2.08464900 & 0.26426700 \\ \mathrm{C} & -2.85117600 & 0.75450100 & -0.27296600 \\ \mathrm{C} & 0.35939900 & 0.63632800 & 0.09128600 \\ \mathrm{C} & -2.02579700 & -0.30593200 & -0.05491900 \\ \mathrm{C} & -0.58250500 & -0.35944700 & 0.06758300 \\ \mathrm{H} & -3.24136400 & 2.81425200 & -0.64123600 \\ \mathrm{H} & -1.12505000 & 3.75426900 & -0.29849200 \\ \mathrm{H} & -3.91114400 & 0.54826000 & -0.40748600 \\ \mathrm{C} & 1.80133800 & 0.26437100 & -0.02005000 \\ \mathrm{O} & 0.89953600 & 2.82274200 & 0.77884300 \\ \mathrm{~S} & -0.32068800 & -2.11706000 & 0.15352900 \\ \mathrm{~S} & -2.47852700 & -2.02570100 & -0.00167400 \\ \mathrm{O} & 2.03322900 & -1.00018100 & 0.38986100 \\ \mathrm{O} & 2.66819200 & 0.97740500 & -0.49548200 \\ \mathrm{H} & 4.40480000 & -0.06021600 & -0.53262300 \\ \mathrm{H} & 2.98826100 & -1.23008800 & 0.20263200\end{array}$




$\begin{array}{llll}\mathrm{O} & 4.64510300 & -0.97226100 & -0.26502300 \\ \mathrm{H} & 5.06002600 & -1.39313200 & -1.03303700\end{array}$

TS67+ $+\mathbf{H}_{2} \mathbf{O}$ (C1) (Figure 2C)

Absolute energy: -1405.6580

Sum of electronic and zero-point Energies $=-1405.5329$

Sum of electronic and thermal Energies $=-1405.5201$

Sum of electronic and thermal Enthalpies $=-1405.5191$

Sum of electronic and thermal Free Energies $=-1405.5726$

Number of Imaginary frequencies: 1

01

$\begin{array}{lrrc}\mathrm{C} & 2.80211100 & 1.48140400 & 0.06742500 \\ \mathrm{C} & 2.99035100 & 0.12690200 & 0.09221300 \\ \mathrm{C} & 2.03597300 & -0.98515800 & 0.25684200 \\ \mathrm{C} & 1.59829100 & 2.25854000 & 0.10048000 \\ \mathrm{C} & 0.61915500 & -0.81898500 & -0.17259700 \\ \mathrm{C} & 0.33258400 & 1.75318900 & 0.00626200 \\ \mathrm{C} & -0.02235000 & 0.37331800 & -0.18683800 \\ \mathrm{H} & 3.71365100 & 2.07665700 & 0.04403500 \\ \mathrm{H} & 4.01827200 & -0.22948800 & 0.09809400 \\ \mathrm{H} & 1.71434300 & 3.33714300 & 0.17437700 \\ \mathrm{C} & -0.25060600 & -2.01731200 & -0.53337500 \\ \mathrm{O} & 2.42439600 & -2.04681900 & 0.73738600 \\ \mathrm{O} & -1.54366200 & -1.69300300 & -0.58119900 \\ \mathrm{O} & 0.18998500 & -3.12345100 & -0.76342900 \\ \mathrm{~S} & -1.78320600 & 0.44781000 & -0.53627200 \\ \mathrm{~S} & -1.21642700 & 2.61740500 & -0.02743800 \\ \mathrm{H} & -2.46912100 & -1.21769800 & 2.34246300 \\ \mathrm{H} & -2.47989000 & -1.81433000 & 0.84448600 \\ \mathrm{O} & -2.90453600 & -1.14243700 & 1.47350800 \\ \mathrm{H} & -2.46563500 & -0.18145700 & 0.80329100\end{array}$

$7+\mathbf{H}_{2} \mathbf{O}(\mathrm{C} 1)$ (Figure 2C)

Absolute energy: -1405.6652

Sum of electronic and zero-point Energies $=-1405.5381$

Sum of electronic and thermal Energies $=-1405.5234$

Sum of electronic and thermal Enthalpies $=-1405.5230$

Sum of electronic and thermal Free Energies $=-1405.5799$

Number of Imaginary frequencies: 0

01

$\begin{array}{lrrc}\mathrm{C} & 2.96355300 & 1.15202200 & 0.21287400 \\ \mathrm{C} & 3.01293000 & -0.21771900 & 0.20652600 \\ \mathrm{C} & 1.96010700 & -1.24622700 & 0.22853700 \\ \mathrm{C} & 1.86250800 & 2.06484400 & 0.17707900 \\ \mathrm{C} & 0.56864700 & -0.87965300 & -0.16729000 \\ \mathrm{C} & 0.54550200 & 1.72179000 & 0.00849800\end{array}$




$\begin{array}{lccc}\mathrm{C} & 0.08388200 & 0.37972500 & -0.19577700 \\ \mathrm{H} & 3.93457300 & 1.64086800 & 0.27755900 \\ \mathrm{H} & 3.99922800 & -0.67139100 & 0.27492300 \\ \mathrm{H} & 2.10240200 & 3.12056000 & 0.27339700 \\ \mathrm{C} & -0.47505400 & -1.92334200 & -0.52281200 \\ \mathrm{O} & 2.23828800 & -2.39687800 & 0.55553600 \\ \mathrm{O} & -1.69396200 & -1.36580900 & -0.70821100 \\ \mathrm{O} & -0.27987800 & -3.10829800 & -0.63985200 \\ \mathrm{~S} & -1.66092200 & 0.55068500 & -0.63193800 \\ \mathrm{~S} & -0.87742600 & 2.75664700 & -0.06646500 \\ \mathrm{H} & -3.14232700 & -0.98012200 & 2.53817700 \\ \mathrm{H} & -3.04375700 & -1.57355000 & 1.09634000 \\ \mathrm{O} & -3.27152900 & -0.77029200 & 1.60024600 \\ \mathrm{H} & -2.31236100 & 0.36791600 & 0.56649400\end{array}$

TS73+ $\mathrm{H}_{2} \mathrm{O}(\mathrm{C} 1)$ (Figure 2C)

Absolute energy: -1405.6580

Sum of electronic and zero-point Energies $=-1405.5336$

Sum of electronic and thermal Energies $=-1405.5208$

Sum of electronic and thermal Enthalpies $=-1405.51988$

Sum of electronic and thermal Free Energies $=-1405.5734$

Number of Imaginary frequencies: 1

$\begin{array}{lrrr}01 & & & \\ \mathrm{C} & 1.11527500 & 2.83172800 & -0.08002500 \\ \mathrm{C} & 2.19647600 & 2.01052800 & 0.09605400 \\ \mathrm{C} & 2.28658200 & 0.56070300 & 0.31195700 \\ \mathrm{C} & -0.28379300 & 2.55010400 & -0.16363000 \\ \mathrm{C} & 1.13972500 & -0.30630100 & -0.07556500 \\ \mathrm{C} & -0.88871900 & 1.31587300 & -0.22695700 \\ \mathrm{C} & -0.14850400 & 0.08327900 & -0.26607200 \\ \mathrm{H} & 1.35202500 & 3.89334500 & -0.13605700 \\ \mathrm{H} & 3.17265000 & 2.48416800 & 0.17315900 \\ \mathrm{H} & -0.94329800 & 3.41357900 & -0.20202800 \\ \mathrm{C} & 1.32122700 & -1.78741400 & -0.23840800 \\ \mathrm{O} & 3.31331100 & 0.07345800 & 0.78098000 \\ \mathrm{O} & 0.10341400 & -2.40944200 & -0.48515700 \\ \mathrm{O} & 2.32932500 & -2.43554300 & -0.19560200 \\ \mathrm{~S} & -1.24428900 & -1.29049400 & -0.64942800 \\ \mathrm{~S} & -2.62453100 & 1.03617800 & -0.35814900 \\ \mathrm{H} & -2.86655600 & -0.14634900 & 1.44974600 \\ \mathrm{H} & -2.12568000 & -0.98006700 & 2.62668300 \\ \mathrm{O} & -2.64349100 & -1.07228200 & 1.80518200 \\ \mathrm{H} & -1.93810800 & -1.39616200 & 0.82237600\end{array}$

$\mathbf{3}+\mathbf{H}_{2} \mathbf{O}(\mathrm{C} 1)$ (Figure 2C)

Absolute energy: -1405.7011 
Sum of electronic and zero-point Energies $=-1405.5756$

Sum of electronic and thermal Energies $=-1405.5604$

Sum of electronic and thermal Enthalpies $=-1405.5595$

Sum of electronic and thermal Free Energies $=-1405.6198$

Number of Imaginary frequencies: 0

01

$\begin{array}{lrrr}\mathrm{C} & 0.75539600 & 2.90317900 & -0.19092300 \\ \mathrm{C} & 1.90924300 & 2.25020300 & 0.10739100 \\ \mathrm{C} & 2.12803000 & 0.83389000 & 0.46793900 \\ \mathrm{C} & -0.58965100 & 2.39833200 & -0.28330600 \\ \mathrm{C} & 1.18884800 & -0.19791800 & -0.02559800 \\ \mathrm{C} & -1.01216800 & 1.10137100 & -0.32172500 \\ \mathrm{C} & -0.14498400 & -0.05306800 & -0.32767800 \\ \mathrm{H} & 0.83681100 & 3.97963000 & -0.33141700 \\ \mathrm{H} & 2.82209000 & 2.83650000 & 0.18829700 \\ \mathrm{H} & -1.36688800 & 3.15590400 & -0.34846200 \\ \mathrm{C} & 1.66456700 & -1.59113100 & -0.20773700 \\ \mathrm{O} & 3.12275300 & 0.53398000 & 1.12150900 \\ \mathrm{O} & 0.60107200 & -2.40536100 & -0.62324500 \\ \mathrm{O} & 2.75858600 & -2.07039200 & -0.08459100 \\ \mathrm{~S} & -0.87522100 & -1.58092600 & -0.80109400 \\ \mathrm{~S} & -2.76573200 & 0.76839200 & -0.56383600 \\ \mathrm{H} & -2.96439200 & 0.13041200 & 0.62180200 \\ \mathrm{H} & -2.89646300 & -0.91766700 & 3.18234400 \\ \mathrm{O} & -2.88727600 & -1.08157900 & 2.22675500 \\ \mathrm{H} & -2.33268200 & -1.86656000 & 2.09271800\end{array}$

Table S1. Computed Entropies, Enthalpies, and Free Energies for 1, 2, and TS12 Reaction<smiles></smiles>

$\begin{array}{cccc}\Delta \mathrm{E}_{\mathrm{rel}}= & \Delta \mathrm{H}_{\text {rel }}= & \Delta \mathrm{S}_{\mathrm{rel}}= & \Delta \mathrm{G}_{\mathrm{rel}}= \\ -1.6 \mathrm{kcal} / \mathrm{mol} & -1.6 \mathrm{kcal} / \mathrm{mol} & 0.4 \mathrm{eu} & -1.7 \mathrm{kcal} / \mathrm{mol}\end{array}$<smiles></smiles>
$\Delta \mathrm{E}^{\ddagger}=$ $\Delta \mathrm{H}^{\ddagger}=$
$9 \mathrm{kcal} /$ $\Delta S^{\ddagger}=$ $\Delta \mathrm{G}^{\ddagger}=$

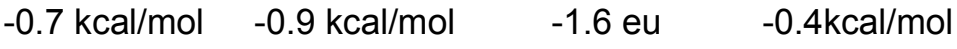

